\title{
Ethische Unbedenklichkeit bestätigen lassen
}

\author{
WISSENSCHAFT ERKLÄRT: ETHIKKOMMISSION Forscher sind vor Beginn \\ einer Patientenstudie dazu verpflichtet, einen Ethikantrag zu stellen, den eine \\ Ethikkommission genehmigen muss. Die Kommission achtet darauf, dass die \\ Wissenschaftler ihre Studienteilnehmer bestmöglich schützen.
}

I m Princeton-Plainsboro Teaching Hospital werden zwei Patienten in lebensbedrohlichem Zustand eingeliefert. Beide haben identische Symptome. Die Diagnose ist unbekannt, die Ärzte sind ratlos. Die einzige Hoffnung ist, dass eines von zwei neuen Medikamenten helfen könnte. Nur: Welches davon ist das richtige? Dr. House entschließt sich in der gleichnamigen Fernsehserie dazu, den zwei Patienten jeweils eines der beiden Medikamente zu verabreichen.

Dies ist ein Beispiel für einen Therapieversuch, ohne dass vorher ein Ethikantrag eingeholt wurde. Aber wann ist ein Ethikantrag eigentlich Pflicht, warum benötigen ihn Forscher und wer ist für seine Überprüfung zuständig?

Patienten und Forscher schützen > Für Wissenschaftler ist es vor der Durchführung einer Patientenstudie verpflichtend, einen sogenannten Ethikantrag zu stellen. Diesen Antrag bearbeitet eine Ethikkommission. Die Kommission hat die Aufgabe, abzuwägen, ob die Untersuchung wichtige humanitäre Verpflichtungen respektiert, beispielsweise die Deklaration von Helsinki von 1964. Das ist eine Erklärung des Weltärztebundes zu den ethischen Grundsätzen bei der Forschung am Menschen. Die Kommission schützt also zum einen die Interessen und Rechte der Probanden, zum anderen bewahrt sie aber auch die Forscher davor, gegen gesetzliche Regelungen zu verstoßen. Die Ethikkommission greift ein, wenn Wissenschaftler gewisse Vorschriften nicht einhalten. So dürfen beispielsweise Probanden erst zur Teilnahme an einer Studie oder einem Experiment einwilligen, wenn man sie ausführlich über deren Nutzen und Risiken informiert hat. Zu dieser Aufklärung gehören Informationen über den Studienablauf, über mögliche Nebenwirkungen der Interventionen und über alternative Behandlungsmöglichkeiten. Auch das Recht auf Teilnahmeverweigerung muss zur Sprache kommen. Die Forscher sind bei Studien verpflichtet, die Probanden mit einer sogenannten Patientenversicherung gegen Schäden abzusichern. Die Teilnahme an einer Studie erfolgt niemals wie bei Dr. House - ohne Information und Zustimmung des Patienten. Zusammengefasst beurteilt eine Ethikkommission also die möglichen Folgen einer Forschungsarbeit aus ethischer, rechtlicher und sozialer Sicht und prüft damit, ob sie ethisch unbedenklich ist.

Das Hinzuziehen einer Ethikkommission ist in Deutschland für jede klinische Prüfung laut Arzneimittelgesetz ( $\$ 40$ Abs. 1 AMG) und Medizinproduktegesetz ( $\S 20$ Abs. 7 MPG) vorgeschrieben. Ethikkommissionen sind bei Landesärztekammern und an medizinischen Fakultäten der Hochschulen eingerichtet. Die zumeist ehrenamtlichen Mitglieder dieser Kommissionen sind in der Regel Mediziner, Naturwissenschaftler oder Statistiker, sie können aber auch Juristen und Theologen sein. Die Zusammenstellung und Verfahrensweise einer Kommission unterscheiden sich je nach Bundesland.

Votum erstellen > Eine Ethikkommission erstellt auf Antrag ein schriftliches Votum für oder gegen das eingereichte Forschungsvorhaben. Sie kann auch ein positives Votum mit Auflagen erstellen, deren Erfüllung die Forscher nachweisen müssen. Eine solche Auflage kann beispielsweise die Verpflichtung zum Abbruch der Studie sein, wenn bei Untersuchungen mit Medikamenten schwere Nebenwirkungen auftreten, bei Zwischenuntersuchungen die Effekte zu gering sind oder wenn der gewünschte Effekt einer Therapie bereits mit 30 statt mit den geplanten 100 Patienten nachweisbar ist.

Ethikanträge müssen verschiedene Punkte beinhalten, zum Beispiel:

> Angaben über den Projektleiter, die Untersucher, die verantwortlichen Statistiker und die teilnehmenden Prüfzentren

> den Beginn und das voraussichtliche Ende der Studie

$>$ die Beschreibung des Versuchsziels

$>$ Angaben zu allen Interventionen und Abweichungen von der Standardbehandlung sowie zu der Art und zum Design der Studie

> Angaben zum potenziellen Nutzen und zu eventuellen Risiken, die die Untersuchung für die Probanden hat

$>$ Angaben darüber, wie wahrscheinlich diese Risiken eintreten werden

$>$ die Ein- und Ausschlusskriterien

$>$ die Abbruchkriterien und die Planung von Zwischenauswertungen

$>$ die statistischen Auswertungsmodalitäten

$>$ die Finanzierung, die Aufwandsentschädigung und die Versicherung

Die Ethikkommmission kann auch Zwischenberichte einfordern. Wie häufig diese erfolgen müssen, hängt von der potenziellen Gefährdung der Patienten ab.

Ethikanträge kosten - je nach Anliegen zwischen 500 und 2.000 Euro. Entscheidend ist, ob die Forscher beispielsweise nur ein Zweitvotum für einen schon genehmigten Antrag benötigen oder ob sie eine große klinische Studie durchführen möchten.

Prof. Dr. Jan Mehrholz

$\Rightarrow$ Ein PDF mit der Deklaration von Helsinki finden Sie unter www.bundesaerztekammer.de/downloads/DeklHelsinki.pdf. 